\title{
Pulse shape discrimination of plastic scintillator EJ 299-33 with radioactive sources
}

\author{
E.V. Pagano ${ }^{a, *}$, M.B. Chatterjee ${ }^{b}$, E. De Filippo ${ }^{c}$, P. Russotto ${ }^{a}$, L. Auditore ${ }^{c, d}$, G. Cardella $^{c}$, \\ E. Geraci ${ }^{\text {c,e }}$, B. Gnoffo ${ }^{\text {c,e }}$, C. Guazzoni ${ }^{\text {f }}$, G. Lanzalone ${ }^{\mathrm{a}, \mathrm{g}}$, S. De Luca ${ }^{\mathrm{d}}$, C. Maiolino ${ }^{\mathrm{a}}$,

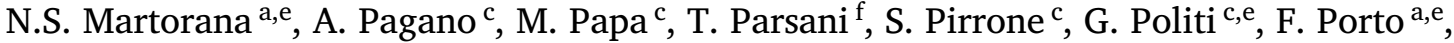

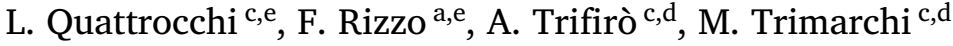 \\ ${ }^{a}$ INFN, Laboratori Nazionali del Sud, Catania, Italy \\ b Saha Institute of Nuclear Physics, Kolkata, India \\ c INFN, Sezione di Catania, Catania, Italy \\ d Dipartimento di Scienze MIFT, Università di Messina, Messina, Italy \\ e Dipartimento di Fisica ed Astronomia, Università di Catania, Catania, Italy \\ ${ }^{\mathrm{f}}$ Dipartimento di elettronica, Informazione, Bioingegneria, Politecnico di Milano e Sezione INFN di Milano, Milano, Italy \\ g Università di Enna "Kore", Enna, Italy
}

ABSTRACT

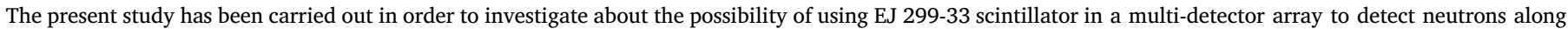

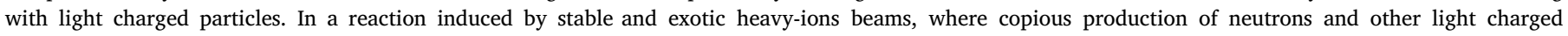

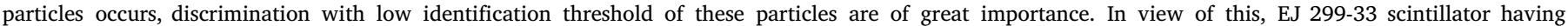

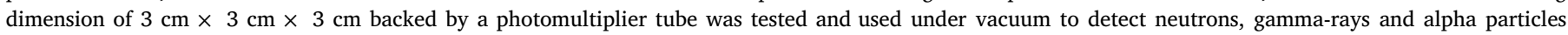

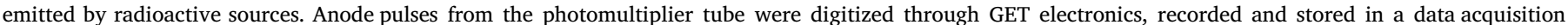

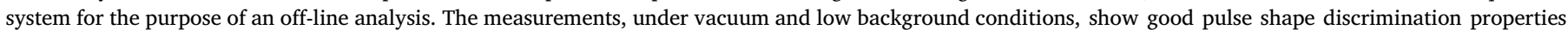

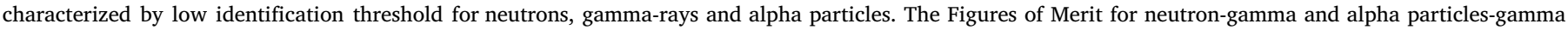
discriminations have been evaluated together with the energy resolution for gamma-ray and alpha particles.

\section{Introduction}

The mechanism of transformation of energy of radiations into light in a scintillator is a complex process. Basic properties of this process were extensively discussed by Birks [1]. For some organic scintillators the main component of light output is very fast and typically of the order of a few ns; as a consequence they can handle high counting rates. The light output of scintillators contains the information (mass, charge and energy) of the impinging radiation. The electrons generated by gamma radiation in the material or the recoiling protons generated by neutrons of similar energy, produce different amounts of light and the energy dependence of the light output is also distinguishable for charged particles of different atomic number even though they have the same energy, due to different ionization densities along the slowing down paths [1]. Typically, there may be two or more time constant decay components in the light output depending on the mode of excitation of the molecular energy levels of the plastic scintillation materials. Fast decay component of the signal arises from the excitation of the singlet states while the slow component arises from more complex mechanism involving triplet states [1]. The fast decay component is not much different for the different interacting particles in the material but slow ones, if any, show dependence on the particle species [1]. Plastic organic scintillators were used earlier in detecting light charged particles in a nuclear reaction and pulse shape discrimination (PSD) of signals were also suggested [1,2]. Alternatively, PSD studies were done with phoswich detectors assembled by two or more scintillators with substantially different time constant decay components. Phoswich detectors were capable of discriminating light ions with atomic number $\mathrm{Z}=1$ up to $\mathrm{Z}=11$ [3]. In projectile-like fragmentation studies at Fermi

* Corresponding author.

E-mail address: epagano@lns.infn.it (E.V. Pagano). 
energy, elemental identification of fast moving particles produced at forward angles, ranging from $\mathrm{Z}=1$ up to the projectile atomic number were reported $[4,5]$. We note that with the advent of doped inorganic crystals such, e.g., CsI(Tl), providing fast and slow decay time components and having high atomic number and good gamma absorption probability, PSD of light isotopes was performed by using single inorganic scintillator. The two primary components of the CsI(Tl) scintillators with decay time constants of $0.68 \mu$ s and $3.34 \mu$ s [2] have been also successfully used in digital acquisition by Amorini et al. [6]. Clusters of a large number of $\mathrm{CsI}(\mathrm{Tl})$ crystals operating under vacuum are widely being used in $4 \pi$ multi-detector arrays [7-10] for full stopping and isotopic discrimination of energetic light charged particles. However, interaction of neutrons with $\mathrm{CsI}(\mathrm{Tl})$ is a rather complicated process and cannot be distinguished easily from gamma-rays. The absorption of neutrons in $\mathrm{CsI}(\mathrm{Tl})$ is due to different processes and reaction Q values, e.g., $\left(n, n^{\prime}-\right.$ gamma $),(n, p),(n, \alpha)$, etc., as recently studied by Auditore et al. [11]. Another disadvantage in using $\mathrm{CsI}(\mathrm{Tl})$ is that the fast decay component of $0.68 \mu$ s is not very suitable for time of flight measurements, requiring time resolution in the range of few nanoseconds. Furthermore, the slow decay time of the fast component also restricts counting rate capability.

Detection of a large number of neutrons along with the charged particles produced in dissipative nuclear reactions is needed to understand the underlying reaction mechanism, especially with radioactive ion beams. The main objective of the present work is to determine a suitable single scintillator that can be used under vacuum with good PSD discrimination properties for charged particles, neutrons and gammarays. A suitable choice is a plastic scintillator, which is rugged, easy to be shaped and machined, and can be used in vacuum. Besides, fast rise-time of a plastic scintillator will be suitable for time of flight measurements with the capability of handling high counting rate. These characteristics also open the possibility to use them in future experiments at medium energies with CHIMERA multi detector [12].

A plastic scintillating system developed in Lawrence Livermore National Laboratory (LLNL), USA, identified by LLNL number 883, was first used for neutrons and gamma-rays pulse shape discrimination by Zaitseva et al. [13]. The Signals collected at the PMT anode were recorded using a digitized pulse to integrate the slow and total components in order to discriminate neutrons from gamma-rays. Hawkes and Taylor [14] observed a good separation of neutrons and gamma-rays on the pulse shape discrimination by using the same plastic scintillator. In particular, they resorted to the fast versus the slow component for the discrimination of neutrons and gamma-rays.

Commercially available plastic scintillators EJ 299-33 [15] from Eljen Technology have been studied [16-20] for $n$-gamma discrimination as a valuable alternative to commonly used liquid scintillators. Pozzi et al. [18] compared the performance of EJ 299-33 plastic scintillator with the EJ 309 liquid scintillator of the same size. In spite of lower light output and scintillation efficiency, EJ 299-33 showed reasonably good PSD properties for neutron and gamma-ray. Discrimination of high energy charged particles were reported by Nyibule et al. [21]. They studied the response of the scintillator to isotopes, from Hydrogen to Carbon, over a wide energy range emerging from the reactions induced by a $240 \mathrm{MeV}{ }^{12} \mathrm{C}$ beam on ${ }^{27} \mathrm{Al}$ target. PSD was studied imposing an online hardware selection by setting two gates on the output signal of the PMT and the charge integration of the two gates were performed by using QDC modules. Kinetic energies of the particles were determined based on the time of flight measurements. Radio-luminescence properties of the scintillator were studied and the dependence of light output with the charged particles up to atomic number $\mathrm{Z}=6$ was reported [21].

The present paper is constructed as follows: In Section 2 the experimental method is described, including information of the read out and digital acquisition. Section 3.1 describes the energy calibration for gamma-rays; Sections 3.2, 3.3 and 3.4 describe the PSD of neutrons and gamma-rays, alphas and gamma-rays and alpha particles, neutrons and gamma-rays, respectively. Section 4 presents the conclusions.

\section{Experimental method}

Experiment to study the pulse shape discrimination of EJ 299-33 having dimension of $3 \mathrm{~cm} \times 3 \mathrm{~cm} \times 3 \mathrm{~cm}$ was carried out inside the CHIMERA [12] scattering chamber under vacuum at INFN Laboratori Nazionali del Sud (LNS), Catania, by using radioactive sources. The scintillation detector was kept unshielded during the measurements. The EJ 299-33 scintillator was optically coupled to a quartz window photomultiplier tube (PMT) 9514B manufactured by EMI operated at a bias of $1.7 \mathrm{KV}$. The emission wavelength of EJ 299-33 peaks at $420 \mathrm{~nm}$ and it matches approximately $70 \%$ of the spectral response of the photo cathode 9514B of the PMT [1]. The anode pulse from the PMT contains the signature of the radiation impinging on the scintillator. Tests were carried out by using an Am-Be neutron source, a mixed-nuclide 3-lines alpha source from AMERSHAM, a Th-alpha source and various gamma sources such as ${ }^{22} \mathrm{Na},{ }^{60} \mathrm{Co}$ and ${ }^{137} \mathrm{Cs}$. Radioactive sources were kept at a distance of 3-4 cm from the scintillator. Neither any moderator for the neutrons nor any absorber for the gamma-rays were used during the tests. Each test run was taken for a long period of time to investigate the long term stability, typically over the night-day cycle, of the electronic systems including the PMT. No noticeable drift of the system was observed during the runs. The output signal from the PMT detector was digitized by GET (General Electronics for TPC) electronics [22,23], which mainly consists of AsAd (ASIC and ADC) board and the AGET chip. The output photomultiplier signal was filtered with the AGET front-end analog filter and shaper (Sallen-Key filter) having a peaking time of $70 \mathrm{~ns}$. The filtered signal $( \pm 1 \mathrm{~V}$ of maximum dynamics) was sampled by the GET at $100 \mathrm{MHz}$ (10 ns-step) through storing in an analog memory based on a Switched Capacitor Array structure and was codified by a 12 bit ADC, allowing off line shape analysis of the detector output. The time off-set (trigger delay) of the digitized signal was about $1 \mu \mathrm{s}$ in order to allow an accurate baseline restoring for each signal. An advantage of using a software analysis is the wider flexibility of gate selection, compared to a hardware one. Selection of gates in the rising part (Fast), in the tailing part (Slow) and on the total part (Total) of the digitized signals has been done through a software. Integrated signals in this three time windows (see Section 3.2) were calculated and stored for the analysis of PSD. The two-dimensional correlation matrices: Slow Vs Fast, Total Vs Slow and Total Vs Fast were analyzed to compare their performances in pulse shape discrimination.

\section{Energy calibration and pulse shape discrimination}

\subsection{Energy calibration for gamma-ray}

The most important measurements for reliable studies of PSD are the pulse height function $\mathrm{L}(\mathrm{E})$ and the resolution $\mathrm{dL}(\mathrm{E}) / \mathrm{L}(\mathrm{E})$, which are characteristics of an individual detector coupled with the pulse processing electronics. The fast electrons, created in gamma-ray interactions with the detector's material, provide clues on the nature of the interaction of the incident gamma-rays. The light output versus electron energy relationship of a particular scintillator depends on several ingredients as the shape, size of the scintillator and its composition, such as the concentration of $\mathrm{H}$ and $\mathrm{C}$ atoms and other constituents as well. In small size plastic scintillators with low atomic number, full energy photopeak gamma-ray absorption is one of the less probable processes; therefore the energy of the impinging gamma-rays is transferred essentially to the recoil electrons through Compton scatterings. For organic scintillator the light output for electrons of energies above $100 \mathrm{keV}$, is assumed to be linear. In order to verify this condition, various gamma-ray radioactive sources $\left({ }^{22} \mathrm{Na},{ }^{60} \mathrm{Co}\right.$ and $\left.{ }^{137} \mathrm{Cs}\right)$ were used for the calibration of the scintillator.

Compton edge energies of $341 \mathrm{keV}$ and $1062 \mathrm{keV}$ corresponding respectively to the $511 \mathrm{keV}$ and $1275 \mathrm{keV}$ gamma-rays of ${ }^{22} \mathrm{Na}$ source, composite Compton edge energies of $1041 \mathrm{keV}$ for gamma-rays of 1173 and $1332 \mathrm{keV}$ of ${ }^{60} \mathrm{Co}$ source, and Compton edge energy of $447 \mathrm{keV}$ of the $662 \mathrm{keV}$ gamma-ray of ${ }^{137} \mathrm{Cs}$ source were used for calibration. 
One such spectrum of Compton profile taken with the 137Cs source is shown in Fig. 1(a).

However, in order to have a good energy calibration, the position of the Compton edge from the Compton distribution, for a gamma-ray of a certain energy, needs to be determined with accuracy. Various prescriptions to determine the position of the Compton edge were given by Knox and Miller [24]. They studied the positions of Compton edges, i.e. the maximum energies in a Compton distribution, for NE 213 liquid scintillators and they leaned towards positioning the Compton edge in correspondence to the $89 \%$ of the maximum of the peak of the Compton distribution.

However, other authors [24] considered different positions of the Compton edge by using a best fit procedures in order to obtain the smallest uncertainty. It may be concluded that the choice of the position corresponding to the maximum electron energy in a Compton distribution is not so unique and it rather depends on the scintillator and electronics. So, in our analysis, positions of the light output (in channels) corresponding to the Compton edge at the peak of Compton distribution and $90 \%, 70 \%$ and $50 \%$ of the maximum beyond the peak of the Compton distribution for each gamma-ray were recorded, in order to determine which one gives the best energy calibration. The best fit was observed with the half-height position (50\%) for the EJ 299-33 scintillator with minimum variance. It may be pointed out that Zaitseva et al. [13] and Pozzi et al. [18] also considered 50\% of the maximum beyond the peak of Compton distribution as the maximum recoil electron energy in their energy calibration with the EJ 299-33 plastic scintillator of different geometry and size. A good linearity of the EJ 299-33 light output versus recoil electron energies is observed in Fig. 2, for the gamma-ray energies of the three radioactive sources used in the tests. The obtained calibration was checked by performing Monte Carlo Simulations of gamma-rays detection with the code MCNPX [25] for all the used sources. In Fig. 1(b) and Fig. 1(c), e.g., such simulations are shown and compared with the experimental spectra for ${ }^{137} \mathrm{Cs}$ and ${ }^{60} \mathrm{Co}$ sources, respectively. The simulated spectra show the characteristic sharp Compton edge profiles. They have been smoothed by applying a convolution of Gaussian energy functions of $25 \%$ (FWHM) resolution, assumed to be a constant value in the investigated range of gamma energy sources, in order to reproduce the experimental Compton profile. A good agreement between simulations and experimental Compton profile was found in all the cases, within the statistical energy accuracy of the calibration, as given by the variances $\sigma_{a}$ and $\sigma_{b}$ of the best fit (see caption of Fig. 2). The dashed (vertical) ridges in Fig. 1(b) and Fig. 1(c) indicate the gamma energy threshold $(\sim 250 \mathrm{kVee})$ adopted during the calibration runs, in order to suppress the low energy background that was dominated by the noise. Above the threshold, the presence of a background is expected in our experimental conditions (see Fig. 1(d) below). Notice that (as previously indicated) our goal was to test discrimination properties and calibrations under realistic conditions in which a large number of potential gamma-ray back scatters were placed in compact configuration close to the plastic (kept unshielded, as mentioned above). Our simulations (made by pointlike source placed in front of the plastic) show that the experimental Compton profile was practically unaffected by the possible persistence of a residual tails of the background well above the adopted threshold. It is right to say that, in view of our experimental conditions, the gammaray resolution used to fit the experimental Compton profile has to be considered as an estimation of an upper limit value. However, we investigated more about the background effects. In Fig. 1(d) we summarize such investigation, e.g, for the ${ }^{60} \mathrm{Co}$ of Fig. 1(c). We assumed an exponential decreasing background having at $0.3 \mathrm{MeV}$ the value given by the difference between the experimental spectrum and the simulated one, as seen in Fig. 1(c), and having at $1.4 \mathrm{MeV}$ a residual tail assumed to be equal to the value of the experimental spectrum (see Fig. 1(d). Consequently, the experimental spectrum of Fig. 1(c) was subtracted by this adopted background and the fitting procedure between the simulated spectrum and the experimental one (subtracted
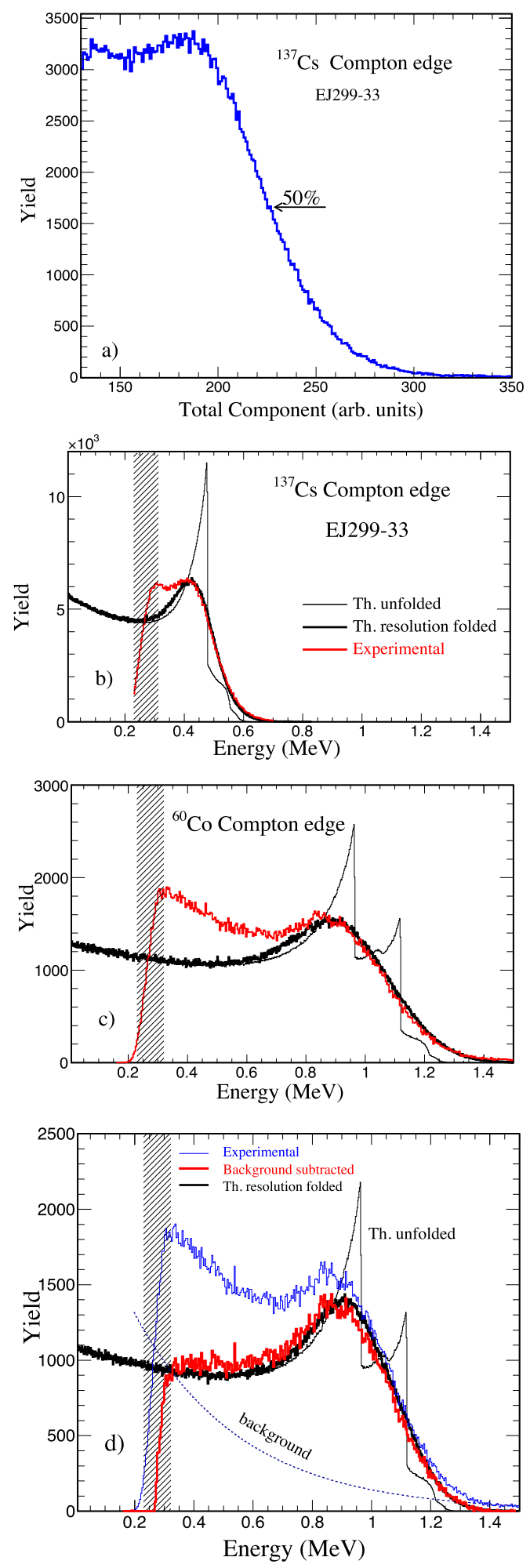

Fig. 1. (a) Gamma spectrum of the uncalibrated light output total component produced by ${ }^{137} \mathrm{Cs}$ source. (b) Gamma spectrum of the calibrated total component produced by ${ }^{137} \mathrm{Cs}$ source compared with the simulated one. (c) Gamma spectrum of the calibrated total component produced by ${ }^{60} \mathrm{Co}$ source compared with the simulated one. Simulated spectra are normalized to the maximum of the Compton peak of the experimental ones. (d) Gamma spectrum of the calibrated total component (thick red line) produced by ${ }^{60} \mathrm{Co}$ source compared with the simulated one (thick black line), after subtraction of the background spectrum, indicated by the dotted line (see text). Simulated spectra are normalized to the maximum of the Compton peak of the (background subtracted) experimental one. (For interpretation of the references to color in this figure legend, the reader is referred to the web version of this article.) 


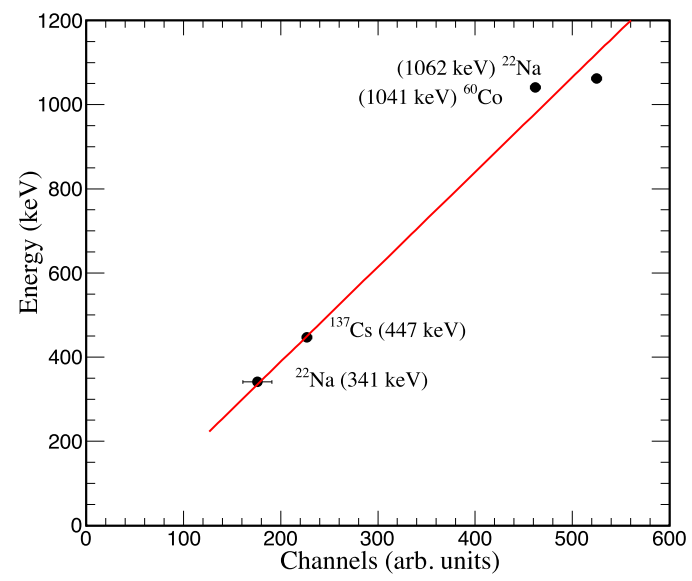

Fig. 2. Energy calibration of the total light output $\left(L_{\mathrm{Tot}}\right)$ of the scintillator with Compton edge energies for the gamma rays of ${ }^{22} \mathrm{Na},{ }^{60} \mathrm{Co}$ and ${ }^{137} \mathrm{Cs}$ sources. $L_{\mathrm{Tot}}=a^{*}$ Channels $+b$; with parameters: $a=2.25 \mathrm{KeV} /$ channel, $b=-61.6 \mathrm{KeV}$, and standard deviations: $\sigma_{a}=0.0487 \mathrm{KeV} /$ Channel; $\sigma_{b}=20.3 \mathrm{KeV}$.

by the background) was repeated again. A good fit (covering practically the full energy range) was found by assuming an overall energy Gaussian resolution convolution of $18 \%$, that includes the electronic noise. Such analysis is in good agreement with the energy resolution obtained for alpha particles (see Section 3.3).

Light output is expressed in terms of electron equivalent energy (keVee). The relation between the equivalent electron energy and proton (neutron) energy of EJ 299-33 depends mostly on the concentrations of $\mathrm{H}$ and $\mathrm{C}$ in that scintillator. Light output produced by neutrons does not show a simple linear dependence on the energy of the detected neutrons, as well as the ones produced by other massive radiations. In order to obtain a functional form of the light output corresponding to the neutron energy, attempts were made to use polynomial functions and power laws by Croft et al. [26] and also rational and exponential forms by Kornilov et al. [27]. Cecil et al. [28] in their experimental studies with neutrons, have parametrized the relation between the total light output $\left(L_{\text {out }}\right)$ and the deposited neutron energy $\left(E_{\mathrm{dep}}\right)$ for hydrocarbon scintillators by the equation:

$L_{\text {out }}=a E_{\text {dep }}-b\left[1-\exp \left(-c E_{\text {dep }}\right)\right]$

In our study we adopted the values of the parameters in Eq. (1) as given by Lawrence et al. [29] for the specific case of EJ 299-33, by applying a small adaptation due to the slight difference in the signal time-integration, reported in Ref. [29], with respect to our case, as they follow: $a=0.8, b=3.9$ and $c=0.22$, of dimensions of $\mathrm{MeVee}^{*} \mathrm{MeV}^{-1}$, MeVee and $\mathrm{MeV}^{-1}$, respectively; consequently, $L_{\text {out }}$ is in MeVee and the $E_{\text {dep }}$ is in $\mathrm{MeV}$.

The Eq. (1) was also used to determine neutron identification threshold measurement in the present work.

\subsection{Pulse shape discrimination of neutrons and gamma-rays}

Pulse shape discrimination of the neutrons and gamma-rays were carried out with a combination of two sources, Am-Be and ${ }^{60} \mathrm{Co}$. Neutron energies spectra from Am-Be source were carefully measured in the past by Notarrigo et al. [30] and more recently by Marsh et al. [31], where neutrons with energy from few hundreds $\mathrm{keV}$ to $10 \mathrm{MeV}$ were observed. Also Am-Be source provides $4.43 \mathrm{MeV}$ gamma-rays; such a high energy gamma-ray has a small deposition of energy in our plastic scintillator. The $60 \mathrm{keV}$ gamma-ray of ${ }^{241} \mathrm{Am}$ source is practically absorbed in the source itself. Therefore, a ${ }^{60} \mathrm{Co}$ source was used along with the Am-Be for neutron and gamma-ray PSD study.

The EJ 299-33 scintillator exhibits three decay components. Depend-ing on the interaction of neutrons or gamma-rays with the scintillator
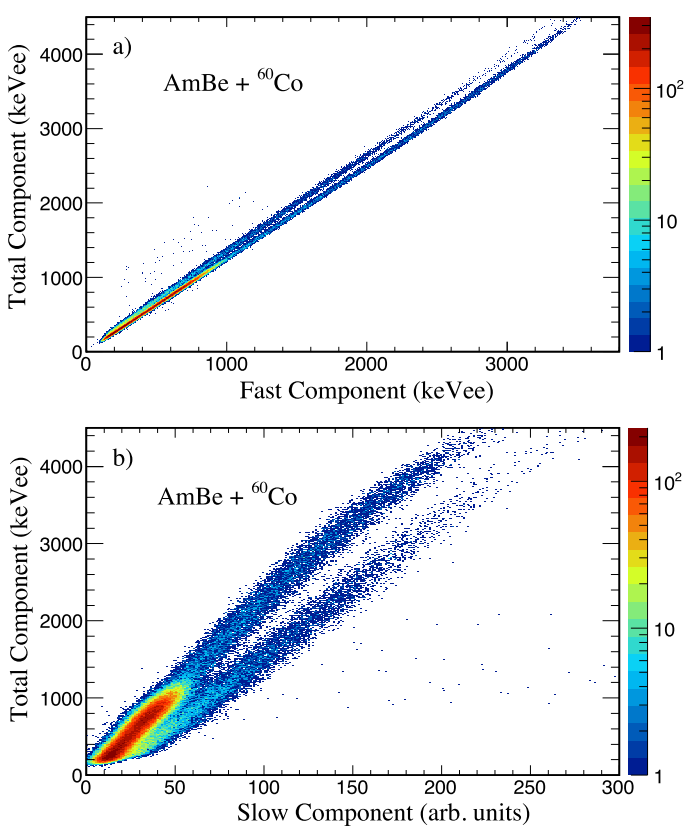

Fig. 3. 2D plots of PSD for n-gamma discrimination taken with Am-Be and ${ }^{60} \mathrm{Co}$ sources (a) Total Vs Fast; (b) Total Vs Slow. (For interpretation of the references to color in this figure legend, the reader is referred to the web version of this article.)

material, the decay components are approximately $13 \mathrm{~ns}, 50 \mathrm{~ns}$ and $460 \mathrm{~ns}$ for neutron excitations and $13 \mathrm{~ns}, 35 \mathrm{~ns}$ and $270 \mathrm{~ns}$ for gammaray excitation [15]. This feature has been utilized in PSD for neutron and gamma-ray separation. The discrimination properties of the scintillator were investigated by comparing the areas under the time windows, selected in different parts of the digitized pulse. In particular, we have used the area of the slow component at various windows in the tail part of the PMT pulse as well as various windows in the fast rising part. The best adopted integration time windows are given in the following. The total integration of digitized pulse was performed in the time range of 30-600 ns. Integration of fast component of the pulse was carried out setting the time window within the range of 30-150 ns. Similarly, integration of slow component was done within the range of 200-600 ns. Total Vs Fast, Total Vs Slow and Slow Vs Fast correlations are compared in Figs. 3(a), 3(b), and 4(a), respectively, where a clear separation of neutrons from gamma-rays is observed. However a better separation was observed in the 2D plots of Slow Vs Fast, starting from an identification threshold value of 150 keVee of the Fast component (Fig. 4(a)). In order to qualify the discrimination capability for a particular value it is common to use a figure-of-merit (FOM) given by the ratio of the difference between the centroids of the gamma-ray and the neutron distribution to the sum of their full widths at half maximum (FWHMs). In our study, the FOM relative to the spectrum of Fig. 4(b) is 1.03. It was obtained by projecting to the Slow component axis of Fig. 4(a) a slice of 1100-1200 keVee of the Fast component (as it is indicated by dashed lines in Fig. 4(a)) that corresponds to neutron energies approximately in the range of $5.0-5.5 \mathrm{MeV}$, as given by Eq. (1). At the threshold, in the range of $150-250 \mathrm{KeVee}$ of the Fast component, the FOM is 0.42 . A low identification threshold as the one reported in this paper by using only PSD discrimination is very important for neutron multiplicity measurements in heavy ion reactions at medium and Fermi energies.

\subsection{Pulse shape discrimination of alphas and gamma-rays}

The analysis of the pulse shape discrimination of alpha particles and gamma-rays has been carried out by using a ${ }^{228} \mathrm{Th}$ source, which has several alpha emissions in its decay chain [32] along with gamma-rays 

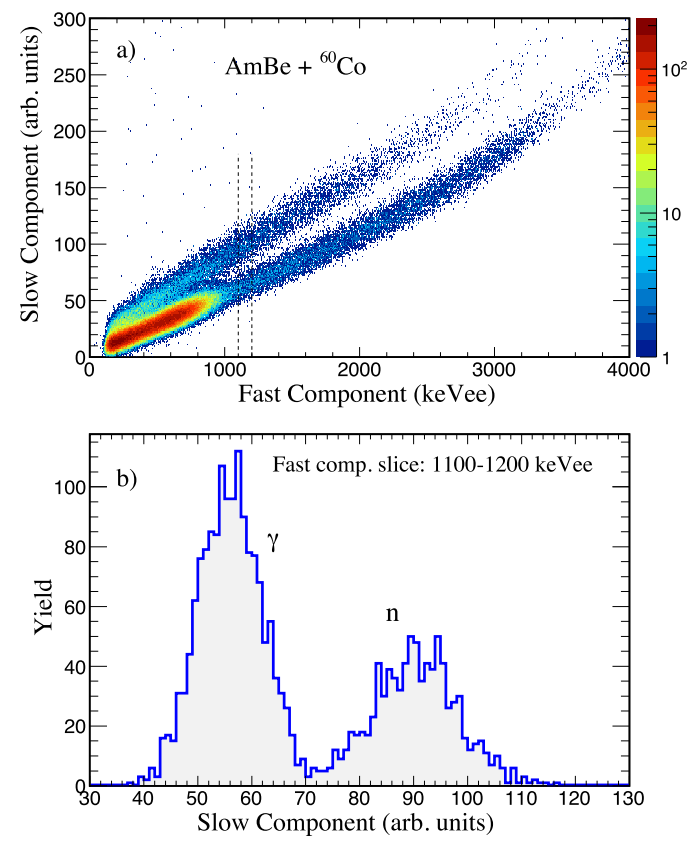

Fig. 4. (a) Slow Vs Fast component 2D plot showing neutron gamma-ray discrimination, (b) Figure of Merit (FOM) for neutron gamma separation relative to the slice in the Fast component shown in Fig. 4(a) with dashed lines.

from daughter nuclei [33]. In particular, ${ }^{228} \mathrm{Th}$-source exhibits alpha particles of energies around 5.4, 5.7, 6.3, 6.8 and $8.7 \mathrm{MeV}$. It has several strong low-energy gamma-rays, with energies ranging between $238 \mathrm{keV}$ to $2614 \mathrm{keV}$. A 2D plot of the PSD of alpha particles and gammarays is shown in Fig. 5(a), where a good separation of alpha particles from gamma-rays is obtained. In Fig. 5(b) a spectrum of the Total component of alpha particles is shown. It was obtained by separating the alpha particles loci against gamma-rays ones by assuming an empirical contour plot in Fig. 5(a) and projected it along with the total component axis. It is observed that lower energy alpha particles between 5.4 and 6.8 $\mathrm{MeV}$ are not resolved and they form a composite peak, while the 8.7 MeV alpha particles are well separated from the composite one. The FWHMs of the composite alpha peaks and of the $8.7 \mathrm{MeV}$ alpha peak (see the full line in Fig. 5(b) were estimated by Gaussian fits. The $8.7 \mathrm{MeV}$ alpha peak showed a total energy resolution of about $18.5 \%$ (FWHM $=166.3 \mathrm{keVee}$ ), which has different contributions from the intrinsic to overall electronic resolution (statistical and transfer contributions of the PMT and the dark noise) [34]. The contribution from the overall electronic resolution was estimated to be about 95.0 keVee. Consequently a value of $15.5 \%$ was estimated for the intrinsic energy resolution of the scintillator for the detection of $8.7 \mathrm{MeV}$ alpha particles.

Notice that this analysis of the energy resolution for alpha particles is independent of the one already discussed for gamma-rays (see Section 3.1). Also, we think useful to give quantitative estimation of the Figure of Merit for the alpha-gamma discrimination property as the one observed in Fig. 5(a). We constrained the event of Fig. 5(a) by a slice around the peak position of the well resolved $8.7 \mathrm{MeV}$ alpha particles in the range 850-950 keVee in the spectrum of Fig. 5(b). The corresponding 2D plot of the PSD discrimination is given as inset in Fig. 6. Consequently, Fig. 6 shows the projected spectrum along with the Slow Component axis and the evaluated value of the FOM $(\alpha-\gamma)=1.09$, corresponding to the two well separated gamma and alpha bumps.

\subsection{Pulse shape discrimination of alpha particles, neutrons and gamma-rays}

Discrimination between alpha particles and neutrons was studied by using ${ }^{228} \mathrm{Th}$ alpha source and Am-Be neutron source. Fig. 7 shows the scatter plot of the Slow component against the Fast one. Two prominent alpha groups are clearly visible along with the recoil protons and
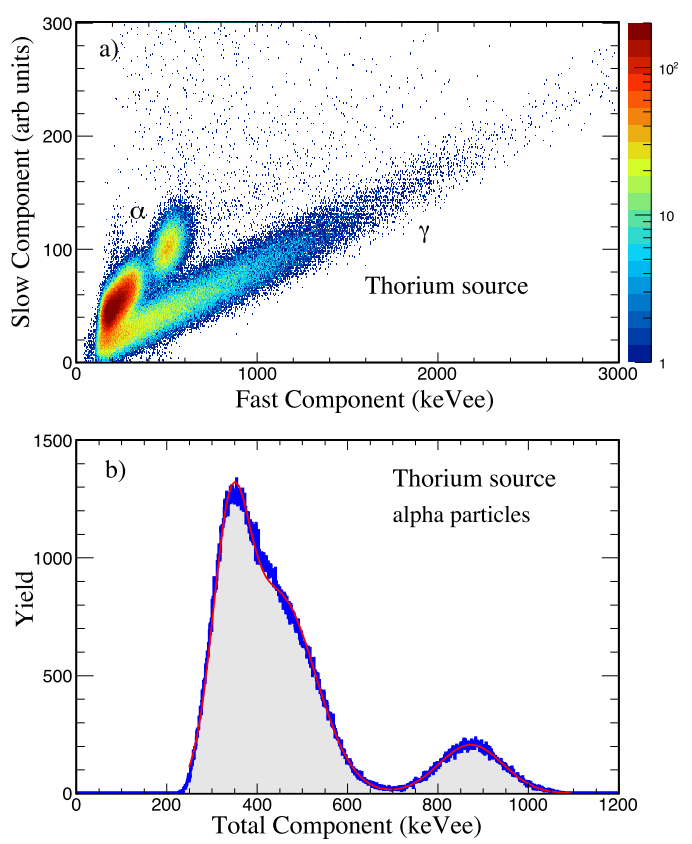

Fig. 5. (a) A 2 D plot of PSD showing alpha-gamma-ray discrimination obtained with ${ }^{228}$ Th source. (b) Total component of alpha particles. The total component group located at lower channels corresponds to composite peak of 4 alpha peaks while the isolated peak at higher channels corresponds to $8.7 \mathrm{MeV}$. The red line is the result of a fit with 3 Gaussians. (For interpretation of the references to color in this figure legend, the reader is referred to the web version of this article.)

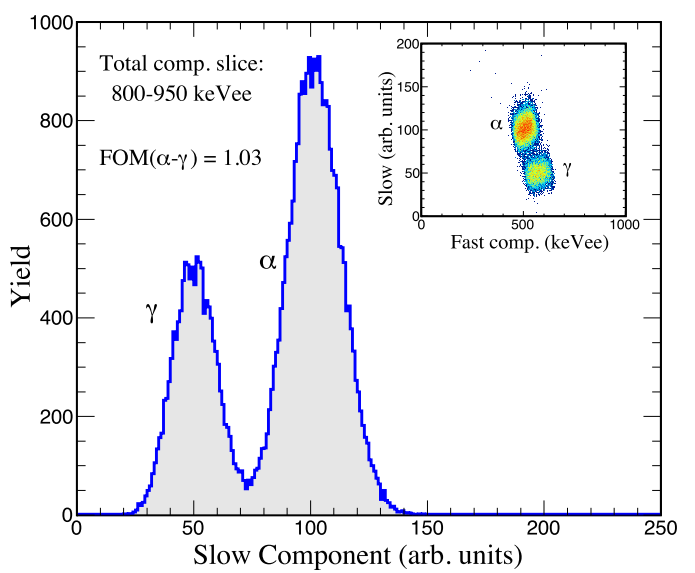

Fig. 6. Figure of Merit (FOM) for $8.7 \mathrm{MeV}$ alpha particles-gamma separation. The inset shows the corresponding Fast vs Slow bidimensional matrix. (For interpretation of the references to color in this figure legend, the reader is referred to the web version of this article.)

gamma-rays. Decay time constants of alpha particles are not given in Ref. [15] for EJ 299-33, but we can argue according to our present PSD analysis and the identification pattern for light ions reported in Ref. [21] that the ratio between the Slow and the Fast time constants of alpha particles should be larger compared to the ones corresponding to the interactions of neutrons or recoil protons. More direct evaluations of the decay time constant of energetic alpha particles can be performed by appropriate measurements with suitable nuclear reactions induced by heavy ion beams.

\section{Conclusions}

In the present work we qualified the performances of the EJ 299-33 scintillator in pulse shape discrimination in presence of alpha particles, 


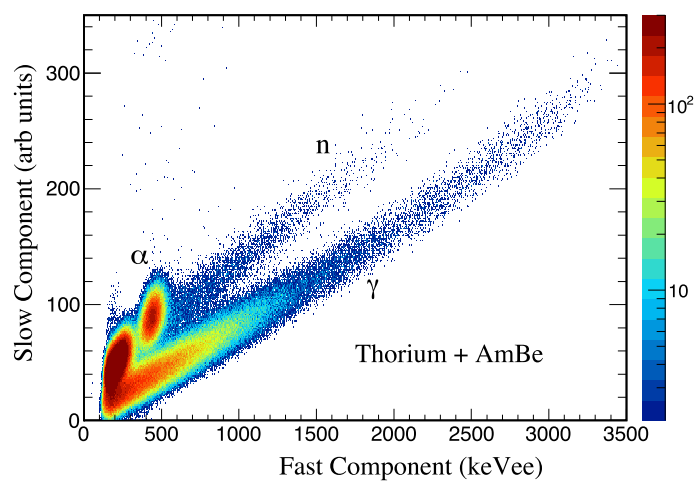

Fig. 7. 2D plot of PSD showing alpha, neutron and gamma-ray discrimination taken with ${ }^{228} \mathrm{Th}$ and $\mathrm{Am}-\mathrm{Be}$ sources.

gamma rays and neutrons. A good pulse shape discrimination has been obtained in the low-energy regime of a few $\mathrm{MeV}$ by using different radioactive sources. Identification threshold of value of $150 \mathrm{keVee}$ of the Fast component corresponding to $1.80 \mathrm{MeV}$ of neutron energy was achieved by using the PSD off-line analysis with digital acquisition. Reasonable good figures of merit were obtained for neutron and gammaray for energy close to the threshold and for $8.7 \mathrm{MeV}$ alpha particlesgamma-ray. From our measurements of the alpha particle spectrum, we have estimated a value of $15.5 \%$ intrinsic resolution of the plastic scintillator for alpha particles of $8.7 \mathrm{MeV}$ energy. The present study suggests also the suitability of EJ 299-33 scintillator for the detection and discrimination of low-energy light ions in heavy ion induced reactions studies. In the next step, we intend to progress in our study of pulse shape discrimination, energy resolutions and Figures of Merit with EJ 299-33 scintillator of light particles and ions produced in reactions induced by medium mass projectile nuclei, where higher backgrounds will be expected.

\section{Acknowledgments}

We are grateful to both Electronics and Advanced Technology services of INFN, Sezione di Catania. Special thanks are due to Mr. Antonio Grimaldi for his valuable assistance and expertise in assembling the PMT/EJ 299-33 detector.

\section{References}

[1] J.B. Birks, The Theory and Practice of Scintillation Counting, Pergamon Press, London, 1964.

[2] G. Knoll, Radiation Detection and Measurements, John Wiley \& Sons, Inc, 2000 and references therein.

[3] C.A. Pruneau, et al., Nucl. Instrum Methods Phys. Res. A 297 (1990) 404.

[4] J.C. Steckmeyer, et al., Nucl. Instrum Methods Phys. Res. A 361 (1995) 472.

[5] G. Lanzanò, et al., Nucl. Instrum Methods Phys. Res. A 323 (1992) 694.

[6] F. Amorini, et al., IEEE Trans. Nucl. Sci. 59 (2012) 1772 and references therein.

[7] R.T. de Souza, et al., Nucl. Instrum Methods Phys. Res. A 295 (1990) 109.

[8] M. Parlog, et al., Nucl. Instrum Methods Phys. Res. A 482 (2002) 674.

[9] M. Alderighi, et al., Nucl. Instrum Methods Phys. Res. A 489 (2002) 257.

[10] S. Wuenschel, et al., Nucl. Instrum. Methods Phys. Res. A 604 (2009) 578.

[11] L. Auditore, A. Pagano, P. Russotto, EPJ Web Conf. 88 (2015) 01001.

[12] A. Pagano, et al., Nucl. Phys. A 734 (2004) 504.

[13] N. Zaitsev, et al., Nucl. Instrum. Methods Phys. Res. A 668 (2012) 88.

[14] N.P. Hawkes, G.C. Taylor, Nucl. Instrum Methods Phys. Res. A 729 (2013) 522.

[15] EJ 299-33 plastic scintillator provisional datasheet, EljenTechnology: http:// www.eljentechnology.com/images/stories/Data_Sheets/Plastic_Scintillators/ ej299-33\%20psd\%20data\%20sheet.pdf.

[16] A.C. Comrie, A. Buffler, F.D. Smit, H.J. Wörtche, in: Proocedings of the conference on technology and instrumentation in particle physics 2014, 2-6 June, 2014, Amsterdam, The Netherlands, Proceedings of Science, PoS, 2014, p. 251.

[17] H. Hartman, A. Barzilov, E.E. Peters, S.W. Yates, Nucl. Instrum. Methods Phys. Res. A 804 (2015) 137.

[18] S.A. Pozzi, M.M. Bourne, S.D. Clarke, Nucl. Instrum. Methods Phys. Res. A 723 (2013) 19.

[19] D. Cester, et al., Nucl. Instrum. Methods Phys. Res. A 735 (2014) 202

[20] A. Buffler, A.C. Comrie, F.D. Smit, H.J. Wörtche, IEEE Trans. Nucl. Sci. 62 (2015) 1442.

[21] S. Nyibule, et al., Nucl. Instrum. Methods Phys. Res. A 728 (2013) 36

[22] J. Giovinazzo, et al., Nucl. Instrum. Methods Phys. Res. A 840 (2016) 15

[23] E.C. Pollacco, et al., Nucl. Instrum. Methods Phys. Res. A 887 (2018) 81.

[24] H.H. Knox, T.G. Miller, Nucl. Instrumn. Methods 101 (1972) 519 and references therein.

[25] D.B. Pelowitz, et al., MCNPX User' s guide, Version 2.5.0, Los Alamos National Laboratory report LA-CP-05-0369 (April 2005).

[26] S. Croft, et al., Nucl. Instrum. Methods Phys. Res. A 316 (1992) 324.

[27] N.V. Kornilov, I. Fabry, S. Oberstedt, F. Hambsch, Nucl. Instrum. Methods Phys. Res. A 599 (2009) 226

[28] R.A. Cecil, B.D. Anderson, R. Madey, Nucl. Instrum. Methods 161 (1979) 439-447.

[29] C.C. Lawrence, et al., Nucl. Instrum Methods Phys. Res. A 759 (2014) 16 and references therein..

[30] S. Notarrigo, F. Porto, A. Rubbino, S. Sambataro, Nuclear Phys. A 125 (1969) 28-32.

[31] J.W. Marsh, D.J. Thomas, M. Burke, Nucl. Instrum. Methods Phys Res. A 366 (1995) 340 and references therein.

[32] https://groups.nscl.msu.edu/hira/pdf/alpha_source_cal.pdf.

[33] W. Maneschg, et al., Nucl. Instrum. Methods Phys Res. A 680 (2012) 161.

[34] M. Moszynski, et al., Nucl. Instrum. Methods. Phys. Res. A 805 (2016) 25-35 and references therein. 